\title{
Melatonin Protects the Esophageal Epithelial Barrier by Suppressing the Transcription, Expression and Activity of Myosin Light Chain Kinase Through ERK1/2 Signal Transduction
}

\author{
Jiacheng Tan ${ }^{a}$ Ying Wang ${ }^{a}$ Yang Xia ${ }^{b}$ Nina Zhang ${ }^{a} \quad$ Xiaomeng Sun ${ }^{a}$ Ting Yu \\ Lin $\operatorname{Lin}^{\mathrm{a}}$ \\ aDepartment of Gastroenterology, The First Affiliated Hospital of Nanjing Medical University, Nanjing, \\ China; 'Department of Thoracic and Cardiovascular Surgery, The First Affiliated Hospital of Nanjing \\ Medical University, Nanjing, China
}

\section{Key Words}

Melatonin • Esophageal epithelial barrier • Myosin light chain kinase

\begin{abstract}
Background/Aims: Dilated intercellular space (DIS) contributes to the pathophysiology of gastroesophageal reflux disease (GERD). Melatonin protects the esophageal mucosa; however, the mechanisms underlying that protection remain unclear. Methods: Transmission electron microscopy (TEM) was used to evaluate the intercellular spaces in the esophageal epithelium of GERD patients. The Het-1A monolayer barrier function was investigated by measuring transepithelial resistance (TER) and FITC-dextran paracellular permeation. The activity of MLCK was represented by MLC phosphorylation. The expression and phosphorylation of MLCK, MLC and ERK were examined by western blot analysis. Results: The expression and activity of MLCK and ERK phosphorylation were increased in the esophageal epithelium. The increased expression and activity of MLCK was correlated with dilated intercellular spaces. Upon acid treatment, the Het-1A monolayer permeability was increased. When the Het-1A monolayer was pretreated with melatonin and PD98059 before the acid incubation, the permeability and the expression and phosphorylation of MLCK and ERK decreased. Conclusion: Melatonin protects the esophageal epithelial barrier by suppressing the transcription, translation and activity of MLCK through ERK1/2 signal transduction. These findings provide a better understanding of the potential clinical application of melatonin in GERD treatment.
\end{abstract}

J. Tan, Y. Wang and Y. Xia contributed equally to this work. 


\section{Introduction}

Gastroesophageal reflux disease (GERD) is one of the most common digestive diseases and can be classified into two different types: reflux esophagitis (RE) and non-erosive reflux disease (NERD). An imbalance between defensive and offensive factors might play an important role in the pathogenesis of GERD [1] . Research studies on epithelial defense have attracted increasing amounts of attention. Dilated intercellular space (DIS) is a feature of the damaged esophageal epithelium and represents increased paracellular permeability [2]. DIS might be an important factor that induces the typical symptoms of GERD by allowing the refluxed nociceptive elements to access the submucosal sensory nerve endings.

Myosin light chain kinase (MLCK) is a $\mathrm{Ca}^{2+}$-calmodulin-dependent serine/threonine kinase that dynamically regulates cellular morphology and contraction [3]. Non-muscle MLCK (nmMLCK), an important member of the MLCK family, is predominantly expressed in endothelial and epithelial cells [4]. According to previous studies, ERK phosphorylation contributes to barrier dysfunction of the vascular endothelium or intestinal epithelium by activating the MLCK signaling pathway $[5,6]$. The role of MLCK in the esophageal epithelial barrier dysfunction has not been elucidated.

Melatonin (MLT), a derivative of tryptophan, has a wide range of biological functions, including immunity enhancement, antioxidation and mucosal protection induced by increased mucosal blood flow [7]. Currently, melatonin has been reported to protect the epithelial and endothelial barrier function through the improvement of paracellular sealing $[8,9]$. Exogenous melatonin has a protective effect on the esophageal mucosa in animal RE models $[10,11]$. Clinical trials have shown that melatonin significantly improves the symptoms of heartburn and abdominal pain in GERD patients $[12,13]$, which suggests that melatonin might have a positive effect on the esophageal epithelial barrier by preventing nociceptive elements from accessing sensory nerve endings. It has been reported that melatonin improves endothelial barrier function by reducing the expression and inhibiting the ERK-mediated kinase activity of MLCK [14]. A similar conclusion was drawn in a study of epithelial MDCK (Madin-Darby canine kidney) cells [15]. The mechanisms underlying melatonin protection of the esophageal epithelium have yet to be elucidated.

This study was designed to investigate the effects of melatonin on the esophageal epithelial barrier and the mechanisms involved in the actions of melatonin.

\section{Materials and Methods}

\section{Participant selection and study design}

A total of 82 subjects were selected for this study, including 59 GERD patients (27 NERD and 32 RE) and 23 controls. The patients who had completed a questionnaire survey and undergone upper gastrointestinal endoscopy were enrolled between February 2014 and May 2014. The specific inclusion criteria and exclusion criteria are shown in Table 1, and a detailed flow chart of patient inclusion is shown in Figure 1A. Subjects with a GERD questionnaire (GerdQ) score $\geq 8$ were considered to have GERD. These patients were further divided into two groups based on their endoscopy results. Patients with mucosal injury and lacking endoscopic or histological evidence of Barrett's esophagus were regarded as having RE and were classified according to the Los Angeles classification. Participants who had a GerdQ score $\geq 8$ and no endoscopic finding were classified as having NERD. The participants without clinical or endoscopic evidence of gastroesophageal reflux served as the control group. Squamous mucosa without erosion was biopsied at $5 \mathrm{~cm}$ above the gastro-esophageal junction for the transmission electron microscopic (TEM), western blot (WB) and real-time quantitative polymerase chain reaction (real-time PCR) analyses. The demographic data and clinical characteristics of the participants are provided in Table 2. The study was approved by the ethics committee of the First Affiliated Hospital of Nanjing Medical University and was performed in accordance with the ethical guidelines of the Declaration of Helsinki. 
Table 1. Participants select. 1 According to the common used GerdQ, typical reflux symptoms were defined as heartburn and regurgitation. 2 Appendectomy is excepted

\begin{tabular}{ll}
\hline Inclusion criteria & Exclusion criteria \\
\hline Age: 18 to 80 & esophageal motility disorders \\
Full sufficiency in literacy & Barrett's esophagus \\
Typical symptoms of GERD 1 & peptic ulcer disease \\
Be off H2-blockers, PPI or antacids $\geq 2$ weeks & gastrointestinal polyps \\
& Gastrointestinal cancer \\
& Gastrointestinal tract surgery 2 \\
& Systemic inflammatory \\
Crohn's disease \\
Ulcerative colitis \\
Stroke \\
Coronary ischemia \\
Acute renal failure \\
A history of H. pylori eradication \\
\hline
\end{tabular}

Table 2. Clinical Characters. pa: Control/NERD; pb: Control/RE; pc: NERD/RE

\begin{tabular}{lcccccc}
\hline Factor & Control & NERD & RE & $\mathrm{p}_{\mathrm{a}}$ & $\mathrm{p}_{\mathrm{b}}$ & $\mathrm{p}_{\mathrm{c}}$ \\
\hline $\mathrm{N}$ & 23 & 27 & 32 & & & \\
$\begin{array}{l}\text { Age (years) } \\
\leq 40\end{array}$ & 10 & 13 & 16 & & & \\
$>40$ & 13 & 14 & 16 & & & \\
Gender & & & & 0.615 & 0.944 & 0.636 \\
Female & 11 & 11 & 15 & & & \\
Male & 12 & 16 & 17 & & & \\
H.pylori & & & & 0.945 & 0.984 & 0.957 \\
$\begin{array}{l}\text { Positive } \\
\text { Negative }\end{array}$ & 13 & 15 & 18 & & & \\
Tobacco & 10 & 12 & 14 & & & \\
Non-smoker & 11 & 14 & 15 & & & \\
Smoker & 12 & 13 & 17 & & & \\
Alcohol & & & & 0.586 & 0.698 & 0.852 \\
Non-drinker & 12 & 12 & 15 & & & \\
Drinker & 11 & 15 & 17 & & & \\
\hline
\end{tabular}

\section{Cell Culture}

The Het-1A cell line (American Type Culture Collection, Manassas, VA, USA), a non-neoplastic esophageal keratinocyte derived cell line, was cultured at $37^{\circ} \mathrm{C}$ in a $5 \% \mathrm{CO}_{2}$-humidified atmosphere in bronchial epithelial cell medium (BEGM BulletKit, Lonza, Walkersville, MD, USA) containing basal medium (BEBM). We used confluent monolayer of Het-1A cells to perform this study.

\section{Transmission electron microscopy (TEM) examination}

The esophageal mucosal biopsies were fixed in a glutaraldehyde solution at $4^{\circ} \mathrm{C}$ and clarified, dehydrated, embedded and sectioned into ultra-thin slices. Ten slices prepared from different sites of the identical biopsy specimen were selected and observed under TEM (2600 J EME2000X, Hitachi, Tokyo, Japan). The Leica image analyzing system (Q550IW, Leica, Wetzlar, Germany) was used to evaluate the intercellular spaces of the esophageal epithelium. Ten images were obtained from each slice. For each image, an intact cell was selected, and the intercellular space was evaluated by determining the vertical distance between the selected cell and its adjacent cells in 10 randomly selected directions. In total, 100 intercellular spaces in 10 images were selected, and the average width of the intercellular space was calculated.

\section{Cell viability}

Confluent Het-1A monolayers were incubated with acidified medium (BEBM, pH 2.0-7.0) for 5, 15, 30 or $60 \mathrm{~min}$. Then, the Het-1A cells were mildly trypsinized and suspended in non-acidic medium; $20 \mu \mathrm{L}$ of the cell suspension was diluted at 1:10 with Trypan blue (2.22 g/L in PBS). The viable cells were counted using a Countess Counter (Invitrogen, Carlsbad, CA, USA). BEBM (pH 4.0) was found to be the optimal acidified medium and was used in the subsequent experiments (Fig. 3A).

Measurement of transepithelial resistance (TER)

TER was measured to assess the barrier function of the confluent Het-1A cell monolayer cultured on Transwell inserts (pore size, $0.4 \mu \mathrm{m}$; PET track-etched membrane, Corning-Costar, Cambridge, UK). The confluent monolayer was incubated in acidified BEBM for 5, 15, 30 or $60 \mathrm{~min}$. After incubation, TER was 
measured using an epithelial volt-ohm meter (Millicell ERS-2 Electrical Resistance System, Millipore, MA, USA) according to the manufacturer's instructions. The monolayer resistance was calculated after subtracting the resistance value of the inserts from the total resistance value and multiplying by the area of the insert. Figure 3B shows that TER is deceased to the minimum level after $30 \mathrm{~min}$ incubation. So we considered 30 min as the optimal incubation duration in acidified BEBM (pH 4.0). To investigate the effects of melatonin on the esophageal epithelial barrier functions and to explore the potential underlying mechanisms, the Het1A monolayer was exposed to various concentrations of melatonin $(0.1,1,10$ or $20 \mu \mathrm{M}$; St. Louis, MO, USA) and PD98059, an ERK inhibitor, (10, 20 or $30 \mu \mathrm{M}$; St. Louis, MO, USA) for different durations before acid exposure. TER is increased to the maximum level after pre-incubating Het-1A monolayer with melatonin $(10 \mu \mathrm{M})$ for $6 \mathrm{~h}$, or PD98059 $(20 \mu \mathrm{M})$ for $2 \mathrm{~h}$ before acid exposure. So we considered those were the optimal concentrations and exposure durations of melatonin and PD98059 (Fig. 3C and D).

\section{Measurement of the epithelial paracellular permeability}

The Het-1A monolayers were incubated with acidified BEBM for $30 \mathrm{~min}$, or with melatonin for $6 \mathrm{~h}$ or PD98059 for $2 \mathrm{~h}$ (according to previous results), followed by incubation with acidified BEBM for $30 \mathrm{~min}$. After the incubations, the media were refreshed, and $1 \mathrm{mg} / \mathrm{mL}$ FITC-dextran $(10 \mathrm{kDa}$, Sigma, St. Louis, Mo., USA) was added to the upper chamber. After $2 \mathrm{~h}$ of incubation at $37^{\circ} \mathrm{C}$, we collected the medium in the lower chamber and measured the fluorescence using a fluorometer (Perkin Elmer Luminescence Counter, MA, USA). The data were calculated as the concentration of FITC-dextran in the lower chamber.

\section{Isolation of the total RNA and quantitative RT-PCR}

Real-time PCR was used to measure the MLCK mRNA expression levels in the esophageal mucosal biopsy tissues and Het-1A monolayer. The biopsy tissues were stored in RNAlater (Ambion, Austin, Texas, USA) at $-20^{\circ} \mathrm{C}$. Total RNA was extracted using TRIzol (Invitrogen, CA, USA) according to the manufacturer's instructions. Then, $2 \mu \mathrm{g}$ of total RNA was reverse transcribed and subjected to realtime PCR using acDNA synthesis kit (New England Biolabs, MA, USA) and a SYBR Green real time PCR Kit (TaKaRa, Dalian, China), respectively. The following MLCK PCR primers were used: forward (5' to 3'), GCATCAAGTACATGCGGCAG; reverse (5' to 3'), GGATGTAGCAGATGACCCCG. $\beta$-actin served as an internal control. The amplification cycle was as follows: denaturation at $95^{\circ} \mathrm{C}$ for $30 \mathrm{sec}$, annealing at 95 $\mathrm{C}$ for $5 \mathrm{sec}$ and extension at $60^{\circ} \mathrm{C}$ for $30 \mathrm{sec}$, repeated 40 times.

\section{Western blot analysis}

The MLCK activity was represented by the MLC phosphorylation level. The expression and phosphorylation of MLCK, MLC and ERK were examined by western blot analysis. Lysis buffer (Beyotime, Shanghai, China) at a concentration of $10 \mathrm{~mL} / \mathrm{g}$ was added to the esophageal mucosal biopsy tissue. The Het$1 \mathrm{~A}$ monolayer was incubated with lysis buffer after removing the culture medium. Protease inhibitor $(1 \mu \mathrm{L} /$ mL; Keygen, Nanjing, China) and phosphatase inhibitor ( $5 \mu \mathrm{L} / \mathrm{mL}$; Keygen, Nanjing, China) were added to the buffer to prevent protein degradation. The proteins were quantified using the bicinchoninic acid (BCA) method. The extracted protein $(30 \mu \mathrm{g})$ was separated by electrophoresis on $10 \%$ sodium dodecyl sulfatepolyacrylamide gels $(100 \mathrm{~V}$ for $1.5 \mathrm{~h})$ and then blotted onto polyvinylidene fluoride (PVDF) membranes, which were then blocked in 5.0\% milk TBST ( $5 \mathrm{~g}$ of milk powder dissolved in $100 \mathrm{~mL}$ of Tris-buffered saline and Tween 20) at room temperature for $1 \mathrm{~h}$. The blots were then incubated with anti-ERK (1:500, Cell Signaling, Boston, MA, USA), phosphorylated ERK (p-ERK) (1:500, Cell Signaling), MLCK (1:5000, Abcam, London, UK), MLC (1:1000, Sigma, St. Louis, Mo., USA) and phosphorylated MLC (p-MLC, 1:1000, Cell Signaling) antibodies overnight at $4^{\circ} \mathrm{C}$. The blots were washed three times with TBST and incubated with horseradish peroxidase-labeled secondary antibodies (1:2000, Bioworld, Beijing, China) at $37^{\circ} \mathrm{C}$ for $2 \mathrm{~h}$. The blots were then stained with Super ECL Plus Detection Reagent (Thermo, PA, USA). The blots were quantified by densitometry using the electrophoresis gel imaging system (Bio-Rad, Hercules, CA, USA). The phosphorylation level of ERK and MLC were calculated as the ratio of the phosphoproteins relative to the total proteins (the absorbance of the phosphoproteins/the absorbance of the total proteins).

\section{Statistical analysis}

The data were analyzed using SPSS, version 18.0, statistical software (SPSS, Inc., Chicago, IL, USA). Comparisons among multiple groups were analyzed by one-way ANOVA. When the homogeneity of variance 


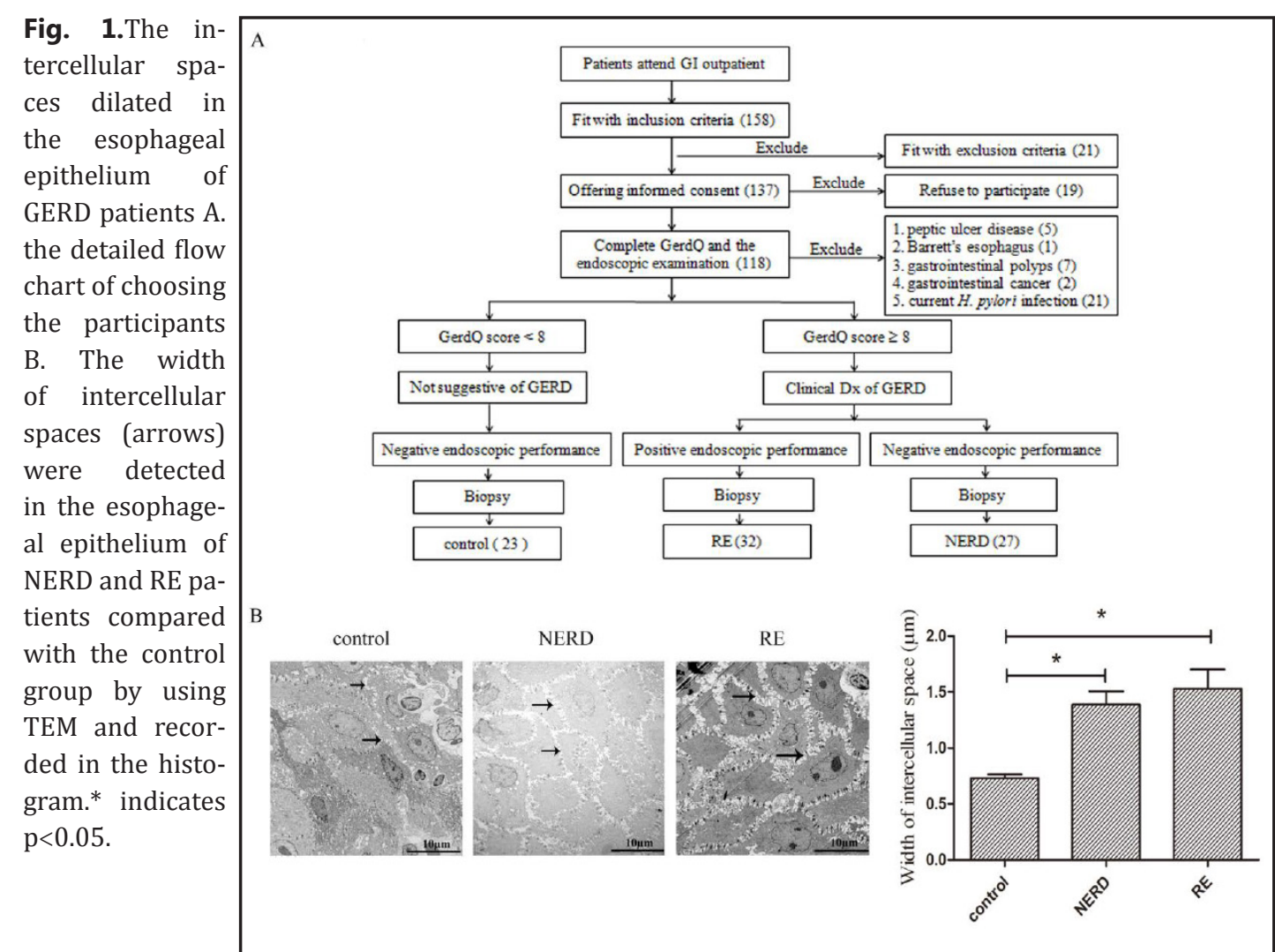

assumption was satisfied, Bonferroni's method was used; otherwise, Tamhane's method was used. The quantitative data were expressed as the mean \pm the standard error of the mean (SEM). The minimal level of significance was identified at $\mathrm{p}<0.05$.

\section{Results}

\section{Demographic data and clinical characters}

In this study, 32 RE patients (median age: 49 years, female: 51.0\%), 27 NERD patients (median age: 48 years, female: $57.2 \%$ ) and 23 non-GERD volunteers (median age: 38 years, female: $43.6 \%$ ) were enrolled according to the screening process (Fig. 1A). The demographic data and clinical characteristics of the selected individuals are shown in Table 2.

The intercellular spaces were dilated in the esophageal epithelium of GERD patients

The intercellular spaces in the esophageal mucosal biopsy tissues were observed using TEM. The ultrastructure of the esophageal epithelial cells was generally intact in all of the subjects. No measurable changes in the intercellular spaces were detected in the epithelium of the control group. The intercellular spaces were dilated dramatically in the RE and NERD groups, but there was no difference between the two groups (Fig. 1B).

The transcription, expression and activity of MLCK as well as the phosphorylation of ERK were upregulated in the esophageal epithelium of GERD patients

The transcription, expression and activity of MLCK (p-/t-MLC) as well as the phosphorylation of ERK (p-/t-ERK) were upregulated in the esophageal mucosal biopsy tissues (Fig. 2A and B). A correlation analysis was conducted to further investigate whether MLCK participates in the modulation of intercellular spaces. The expression and activity of MLCK (p-/t-MLC) was positively correlated with the intercellular spaces in the NERD and RE groups (Fig. 2C). 
Fig. 2. The transcription, expression and activity of MLCK were up-regulated as well as the phosphorylation of ERK in the esophageal epithelium of GERD patients. A. MLCK mRNA expression in the NERD $(n=27)$ and RE $(n=32)$ patients compared with the control group $(n=23)$ relative to $\beta$-actin was detected by using qRT-PCR $(\mathrm{P}<0.05)$. B. The phosphorylation levels of ERK (p-/tERK) in the NERD and RE patients compared with the control group were analyzed by using western-blotting. $\beta$-actin was used as a control. The expression and activity of MLCK in the NERD and RE patients compared with the control group were analyzed by using western-blotting. The activity of MLCK was represented by the phospho-

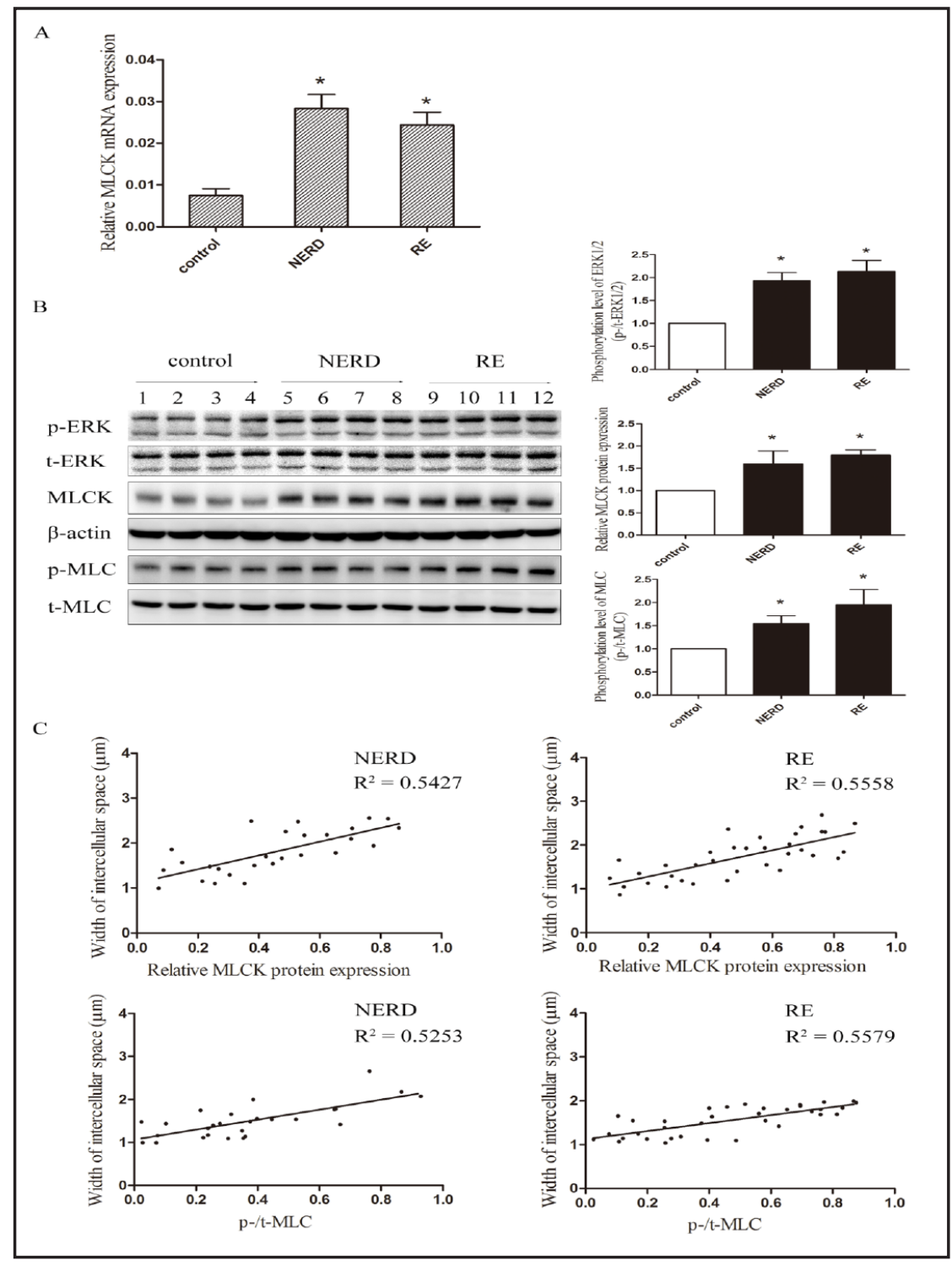
rylation of MLC (p-/t-MLC). All experiments were performed in triplicate and the band intensity values were analyzed by using Image J. C. A positive correlation was found between the expression of MLCK and the intercellular spaces in NERD $\left(R^{2}=0.5427 ; p<0.0001\right)$ and $R E\left(R^{2}=0.5558 ; p<0.0001\right)$. A positive correlation was found between the activity of MLCK and the intercellular spaces in NERD (R2 $=0.5253 ; \mathrm{p}<0.0001)$ and $\operatorname{RE}\left(\mathrm{R}^{2}=0.5579 ; \mathrm{p}<0.0001\right) . *$ indicates $\mathrm{p}<0.05$.

\section{Melatonin improved the barrier function of the Het-1A monolayer against acid}

We sought to explore the effects of melatonin on the esophageal epithelial barrier function in vitro. Het-1A monolayer was incubated in acidified medium to simulate GERD. The barrier function of the Het-1A monolayer was noticeably decreased after incubation with BEBM (pH 4.0) for 30 min (Fig. 3A and B). To further verify the effect of melatonin on the barrier function of the acid-treated Het-1A monolayer, the monolayer was pretreated with melatonin prior to acid exposure. The protective effect of melatonin on the esophageal epithelial barrier was detected by examining the cell's morphology by TER as well as by measuring the FITC-dextran paracellular flux of the Het-1A monolayer. Significant effects of melatonin $(10 \mu \mathrm{M})$ on the barrier functions were observed after a 6-h incubation, whereas shorter incubation times (lower incubation concentration) failed to significantly 
Fig. 3. Melatonin improved the barrier function of Het-1A monolayers against acid. A. Effects of acidified medium on the viability of Het-1A cells. Acidified medium ( $\mathrm{pH} 2.0$ or 3.0) induced significant cellular injury in a time- and $\mathrm{pH}$-dependent fashion, while acidified medium at $\mathrm{pH}$ 4.0-7.0 did not affect the viability of Het-1A cells. B. Effects of acidified medium at $\mathrm{pH} 4.0$ on transepithelial resistance (TER) of Het-1A monolayer. Acidified medium $(\mathrm{pH} \mathrm{4.0)}$ reduced TER in a time-dependent manner. After incubation for 30 minutes, TER decreased to the lowest level. * indicated $\mathrm{p}<0.05$ vs. control (normal conditions). *\# indicated $\mathrm{p}<0.05$ vs. incubation for 15 minutes. C. Effects of melatonin (MLT) on TER of Het-1A monolayer in the presence of acid. MLT pro-
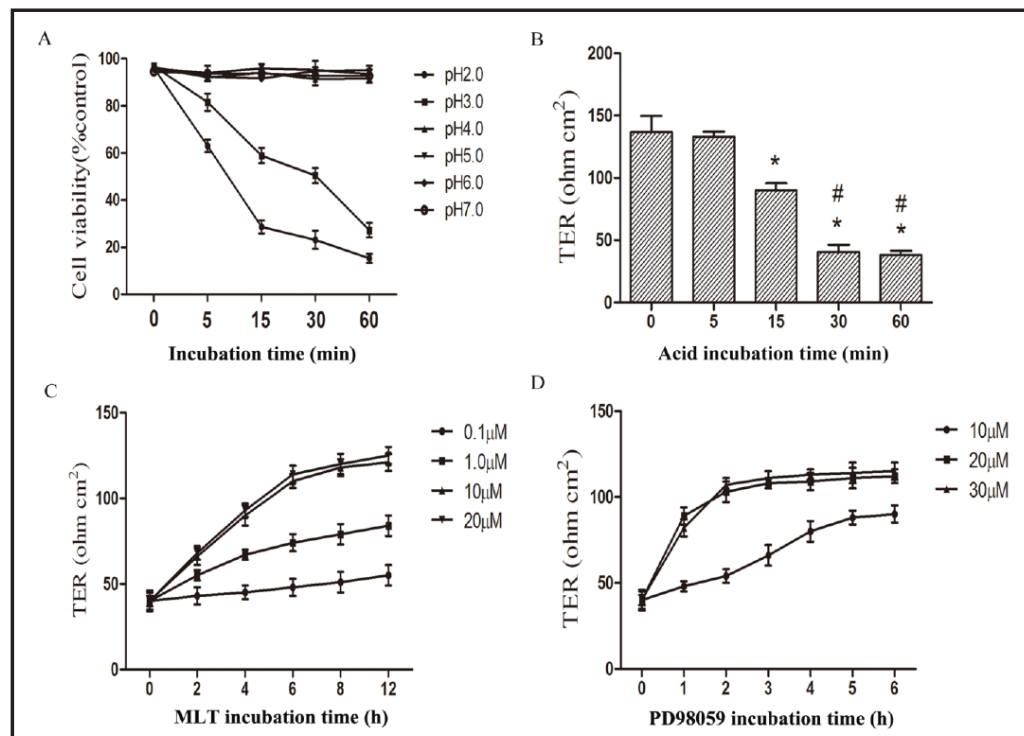

E

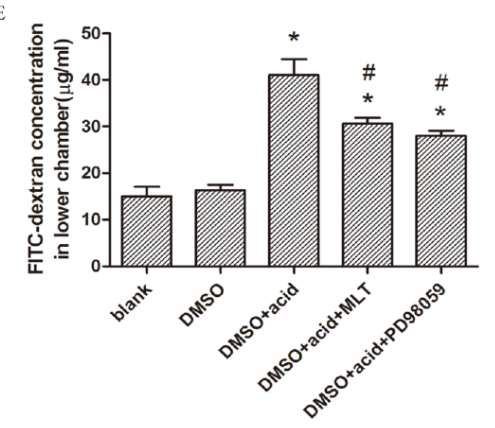

tected Het-1A monolayer barrier in a time- and concentration-dependent manner. Acid-induced reduction of TER was prevented via pre-incubating Het-1A monolayer with MLT $(10 \mu \mathrm{M})$ for $6 \mathrm{~h}$ before acid exposure. D. Effects of PD98059 on TER of Het-1A monolayer in the presence of acid. PD98059 protected Het-1A monolayer barrier in a time- and concentration-dependent manner. Acid-induced reduction of TER was prevented via pre-incubating Het-1A monolayer with PD98059 $(20 \mu \mathrm{M})$ for $2 \mathrm{~h}$ before acid exposure. Values are means \pm SEM of each of 8 experiments. E. Effects of MLT and PD98059 on acid-induced increase in paracellular flux of FITC-dextran. MLT and PD98059 reversed the action of acid significantly. * indicated p $<0.05$ vs. blank. *\# indicated $\mathrm{p}<0.05$ vs. DMSO+acid.

downregulate the barrier functions (Fig. $3 \mathrm{C}$ and $\mathrm{E}$ ). We used melatonin $(10 \mu \mathrm{M})$ in a 6-h incubation time in all of the subsequent experiments.

Melatonin protected the Het-1A monolayer barrier by downregulating the transcription, expression and activity of MLCK through ERK signal transduction

The phosphorylation of ERK was upregulated in the esophageal epithelium of GERD patients, and the intercellular space was positively correlated with the expression and activity of MLCK. We hypothesized that ERK and MLCK might participate in the protective effect of melatonin on the esophageal epithelial barrier. To test this hypothesis, we investigated the expression and activity of MLCK (as determined by MLC phosphorylation) in response to melatonin and/or PD98059 in acid-treated Het-1A monolayers.

First, the Het-1A monolayer was pretreated with PD98059 prior to acid exposure. The protective effect of PD98059 on the esophageal epithelial barrier was detected by examining the cell's morphology using TER and measuring the FITC-dextran paracellular flux of the Het-1A monolayer. Significant effects of PD98059 $(20 \mu \mathrm{M})$ on the barrier functions were 
Fig. 4. Melatonin protected Het-1A monolayers barrier via down-regulating the transcription, expression and activity of MLCK through ERK signal transduction. A. Effects of MLT and PD98059 on the transcription of MLCK relative to GAPDH was detected by using qRT-PCR $(\mathrm{P}<0.05)$. Acid incubation up-regulated the transcription of MLCK significantly, MLT and PD98059 reversed the action of acid. * indicated $\mathrm{p}<0.05$ vs. blank. *\# indicated p $<0.05$ vs. DMSO+acid. B. Effects of MLT and PD98059 on the phosphorylation of ERK (p-/t-ERK) and the expression and activity of MLCK were analyzed by using western-blotting. The phosphorylation of MLC was used to represent the activity of MLCK (p-/t-MLC). Acid incubation promoted the expression and activity of MLCK as well as

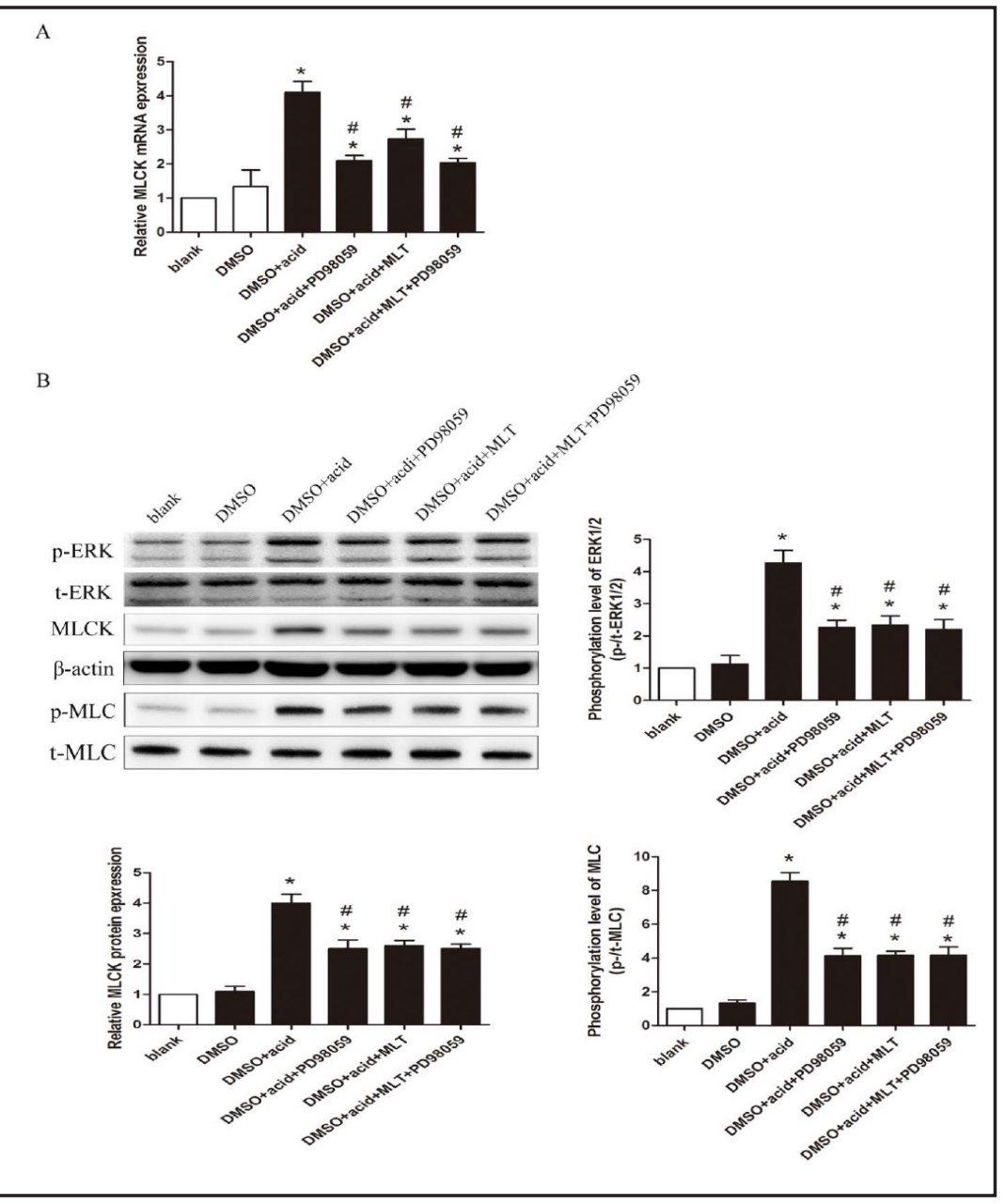
the phosphorylation of ERK. MLT and PD98059 reversed the action of acid. * indicated p < 0.05 vs. blank. *\# indicated $p<0.05$ vs. DMSO+acid. $\beta$-actin was used as a control. All experiments were performed in triplicate and the band intensity values were analyzed by using Image J. C.

observed after a 2-h incubation, whereas shorter incubation times (lower incubation concentration) failed to significantly downregulate the barrier functions. So we used $20 \mu \mathrm{M}$ of PD98059 with a 2-h incubation time in all of the subsequent experiments (Fig. 3D and E).

The transcription, expression and activity of MLCK in the Het-1A monolayer were upregulated after incubation in acid. Melatonin reversed the acid-induced increases in MLCK expression and activity (Fig. 4A and B). Additionally, the increase in ERK phosphorylation in the acid-treated Het-1A monolayer was reversed by pretreatment with melatonin (Fig. 4B). To explore whether acid-induced ERK activation is upstream of MLCK expression and activation, the Het-1A monolayer was pretreated with PD98059, an ERK inhibitor, and challenged with melatonin, before the acid incubation. The expression and activity of MLCK were reversed by pretreatment with PD98059 in the acid-treated Het-1A monolayer (Fig. 4A and $B$ ). These results indicated that ERK activation is an upstream event of MLCK expression and activation. In addition, the MLCK expression and activity did not change significantly in the acid-PD98059-melatonin-treated Het-1A monolayer compared with the acid-PD98059treated and acid-melatonin-treated Het- $1 \mathrm{~A}$ monolayer. These findings suggest that melatonin has a protective effect on the Het-1A monolayer barrier by downregulating the transcription, expression and activity of MLCK through ERK signal transduction. 


\section{Discussion}

GERD is a widespread disorder caused by the reflux of acid and other gastric contents from the stomach into the esophagus [16]. The major symptoms of GERD are acid reflux and heartburn. One-third of GERD patients with endoscopic evidence of esophageal mucosal damage exhibit reflux esophagitis (RE), whereas the patients that only exhibit GERD symptoms are defined as having non-erosive reflux disease (NERD).

Multiple mechanisms lead to GERD, including a mechanically defective lower esophageal sphincter, increased sensitivity of the esophageal mucosa to noxious reflux, and an imbalance between the defensive and offensive factors in the esophageal epithelium. The defensive barriers of the esophageal epithelium include pre-epithelial, epithelial and post-epithelial defenses. The integrity of the esophageal epithelial defense is based on the epithelial cells and paracellular sealing. The esophageal epithelial cells serve as the cellular barrier, whereas the intercellular spaces account for the paracellular sealing. Esophageal epithelial barrier function could be represented by paracellular sealing. In 1996, Tobey et al. first reported that the intercellular spaces were dilated in esophageal mucosa biopsy specimens from GERD patients [2]. A widely accepted view is that dilated intercellular spaces (DIS) allow nociceptive elements in the esophageal contents to access the sensory nerve endings in the esophageal mucosa, which might be an important factor in inducing the heartburn symptoms of GERD.

MLCK is a family of soluble protein kinases encoded by the mylk1-3 genes [17]. There are several MLCK isoforms, including cardiac MLCK (cMLCK), skeletal MLCK (skMLCK), smooth muscle MLCK (smMLCK) and non-muscle MLCK (nmMLCK). NmMLCK, previously known as endothelial MLCK (eMLCK), is predominantly distributed in endothelial and epithelial tissues, including the gut epithelium $[18,19]$.

Emerging studies suggested that the activation of MLCK mediates endothelial and epithelial barrier dysfunction [20,21], and MLCK catalyzes MLC phosphorylation and triggers the contraction of the actin cytoskeleton. The intercellular junction proteins anchored to the actin cytoskeleton are subsequently redistributed, leading to DIS and endothelial/epithelial barrier dysfunction [22, 23]. This mechanism is considered to be the major mechanism of DIS formation in response to factors that induce endothelial and epithelial hyperpermeability. Additionally, MLCK activation causes barrier dysfunction through the phosphorylation and downregulation of the intercellular junction proteins [24]; however, the mechanisms are poorly understood. Prior studies have demonstrated that ERK phosphorylation contributes to the activation of the MLCK signaling pathway, which leads to barrier dysfunction in the vascular endothelium or intestinal epithelium [6]. Although MLCK is implicated in endothelial and epithelial barrier dysfunction $[25,26]$, its specific role in the impairment of the esophageal epithelial barrier has not been reported. Our study provides direct evidence that ERK-mediated activation of MLCK plays a critical role in esophageal epithelial barrier dysfunction.

Melatonin (MLT), originally discovered in the pineal gland, is expressed in all segments of the gastrointestinal tract (GIT), including the esophagus [27]. It is a versatile and ubiquitous hormonal molecule with multiple biological functions, including regulation of the circadian cycles [28] and reproductive rhythms [29] as well as anti-oxidative [30] and antiinflammatory functions [31]. Melatonin has been shown to protect the barrier function in some types of endothelial and epithelial cells $[8,9]$. Animal and clinical studies have shown that melatonin protects the esophageal epithelium of RE/GERD without any side effect [1013]. The underlying molecular mechanisms remain unclear and should be elucidated. In this study, we investigated the protective effect of melatonin on the esophageal epithelial barrier and the underlying mechanisms by which melatonin protects the esophageal epithelial barrier.

The Het-1A monolayer barrier function was improved by melatonin based on the permeability assay results in this study. Some previous studies have shown that melatonin improves the epithelial or endothelial barrier function by inhibiting ERK activation. A 
range of studies has demonstrated that MLC phosphorylation, which is mediated by the upregulation of MLCK expression and activity, is required for epithelial barrier disruption [32]. Additionally, we confirmed that acid injures the barrier function of the Het-1A monolayer via the activation of the ERK/MLCK pathway. These findings prompted us to consider that melatonin might protect the esophageal epithelial barrier through the ERK/ MLCK pathway, and our results confirmed this hypothesis. Melatonin downregulated the phosphorylation of ERK and protected the Het-1A monolayer barrier by suppressing the transcription, expression and activation of MLCK. Thus, melatonin protects the esophageal epithelial barrier by downregulating ERK/MLCK signal transduction.

Some hyperpermeability-associated factors, such as phosphorylation or decreased expression of cell junction proteins or cytoskeletal adapter proteins, should be further investigated to determine whether these events are physiologically important for esophageal epithelial barrier dysfunction. Additionally, based on this study, it is difficult to determine the specific mechanisms by which melatonin downregulates the ERK/MLCK signaling pathway. Because the contents of the stomach reflux contain acid and bile acid, further research is required to determine the effects of bile acid on the esophageal epithelial barrier function.

In our study, we demonstrated that melatonin exerts beneficial effects on the esophageal epithelial barrier by downregulating the ERK/MLCK pathway. Because the side effects of melatonin are remarkably limited, more elaborate studies are necessary to investigate melatonin as a treatment for esophageal barrier dysfunction in GERD.

\section{Acknowledgments}

This work was supported by the Natural Science Funds of China (No. 81270462) and the Postgraduates' Innovation Program of Jiangsu Province (No. Jx22013279).

\section{Disclosure Statement}

The authors have no conflicts of interest to declare.

\section{References}

1 Orlando RC: Current understanding of the mechanisms of gastro-oesophageal reflux disease. Drugs 2006;66 Suppl 1:1-5; discussion 29-33.

2 Tobey NA, Carson JL, Alkiek RA, Orlando RC: Dilated intercellular spaces: A morphological feature of acid reflux--damaged human esophageal epithelium. Gastroenterology 1996;111:1200-1205.

3 Chen D, Lin Y, Xiong Y: Epithelial mlck and smooth muscle mlck may play different roles in the development of inflammatory bowel disease. Dig Dis Sci 2014;59:1068-1069.

-4 Rigor RR, Shen Q, Pivetti CD, Wu MH, Yuan SY: Myosin light chain kinase signaling in endothelial barrier dysfunction. Med Res Rev 2013;33:911-933.

-5 Al-Sadi R, Guo S, Ye D, Ma TY: Tnf-alpha modulation of intestinal epithelial tight junction barrier is regulated by erk1/2 activation of elk-1. Am J Pathol 2013;183:1871-1884.

-6 Wu MH, Yuan SY, Granger HJ: The protein kinase mek1/2 mediate vascular endothelial growth factor- and histamine-induced hyperpermeability in porcine coronary venules. J Physiol 2005;563:95-104.

7 Bubenik G: Thirty four years since the discovery of gastrointestinal melatonin. J Physiol Pharmacol 2008;59:33-51.

8 Sommansson A, Nylander 0, Sjöblom M: Melatonin decreases duodenal epithelial paracellular permeability via a nicotinic receptor-dependent pathway in rats in vivo. J Pineal Res 2013;54:282-291.

-9 Yuan X, Li B, Li H, Xiu R: Melatonin inhibits il-1ß-induced monolayer permeability of human umbilical vein endothelial cells via rac activation. J Pineal Res 2011;51:220-225. 
10 Konturek PC, Brzozowska I, Targosz A, Pawlik M, Kania J, Hess T, Kwiecien S, Konturek SJ, Reiter RJ, Brzozowski T: Esophagoprotection mediated by exogenous and endogenous melatonin in an experimental model of reflux esophagitis. J Pineal Res 2013;55:46-57.

11 Singh P, Singh N, Ahmad A, Singh O, Palit G: Melatonin independent protective role of l-tryptophan in experimental reflux esophagitis in rats. Pharmacol Res 2011;64:249-257.

12 Kandil TS, Mousa AA, El-Gendy AA, Abbas AM: The potential therapeutic effect of melatonin in gastroesophageal reflux disease. BMC Gastroenterol 2010;10:7.

13 Pereira RdS: Regression of gastroesophageal reflux disease symptoms using dietary supplementation with melatonin, vitamins and aminoacids: Comparison with omeprazole. J Pineal Res 2006;41:195-200.

14 Zhu H-Q, Cheng X-W, Xiao L-L, Jiang Z-K, Zhou Q, Gui S-Y, Wei W, Wang Y: Melatonin prevents oxidized low-density lipoprotein-induced increase of myosin light chain kinase activation and expression in huvec through erk/mapk signal transduction. J Pineal Res 2008;45:328-334.

15 Soto-Vega E, Meza I, Ramírez-Rodríguez G, Benitez-King G: Melatonin stimulates calmodulin phosphorylation by protein kinase c. J Pineal Res 2004;37:98-106.

16 Vakil N, van Zanten SV, Kahrilas P, Dent J, Jones R, Global Consensus G: The montreal definition and classification of gastroesophageal reflux disease: A global evidence-based consensus. Am J Gastroenterol 2006;101:1900-1920; quiz 1943.

17 Lazar V, Garcia JG: A single human myosin light chain kinase gene (mlck; mylk). Genomics 1999;57:256267.

18 Garcia JG, Lazar V, Gilbert-McClain LI, Gallagher PJ, Verin AD: Myosin light chain kinase in endothelium: Molecular cloning and regulation. Am J Respir Cell Mol Biol 1997;16:489-494.

19 Shen Q, Rigor RR, Pivetti CD, Wu MH, Yuan SY: Myosin light chain kinase in microvascular endothelial barrier function. Cardiovasc Res 2010;87:272-280.

20 Turner JR, Rill BK, Carlson SL, Carnes D, Kerner R, Mrsny RJ, Madara JL: Physiological regulation of epithelial tight junctions is associated with myosin light-chain phosphorylation. Am J Physiol Cell Physiol 1997;273:C1378-C1385.

21 Reynoso R, Perrin RM, Breslin JW, Daines DA, Watson KD, Watterson DM, Wu MH, Yuan S: A role for long chain myosin light chain kinase (mlck-210) in microvascular hyperpermeability during severe burns. Shock 2007;28:589-595.

22 Ivanov AI, Naydenov NG: Dynamics and regulation of epithelial adherens junctions: Recent discoveries and controversies. Int Rev Cell Mol Biol 2013;303:27-99.

23 Hu YJ, Wang YD, Tan FQ Yang WX: Regulation of paracellular permeability: Factors and mechanisms. Mol Biol Rep 2013;40:6123-6142.

24 Al-Sadi R, Ye D, Said HM, Ma TY: Cellular and molecular mechanism of interleukin-1beta modulation of caco-2 intestinal epithelial tight junction barrier. J Cell Mol Med 2011;15:970-982.

25 Beard RS, Haines RJ, Wu KY, Reynolds JJ, Davis SM, Elliott JE, Malinin NL, Chatterjee V, Cha BJ, Wu MH, Yuan SY: Non-muscle mlck is required for $\beta$-catenin- and foxo1-dependent downregulation of cldn5 in il-1 $\beta$ mediated barrier dysfunction in brain endothelial cells. J Cell Sci 2014;127:1840-1853.

26 Luo HM, Du MH, Lin ZL, Zhang L, Ma L, Wang H, Yu W, Lv Y, Lu JY, Pi YL, Hu S, Sheng ZY: Valproic acid treatment inhibits hypoxia-inducible factor 1alpha accumulation and protects against burn-induced gut barrier dysfunction in a rodent model. PLoS One 2013;8:e77523.

27 Konturek SJ, Konturek PC, Brzozowska I, Pawlik M, Sliwowski Z, Czesnikiewicz-Guzik M, Kwiecien S, Brzozowski T, Bubenik GA, Pawlik WW: Localization and biological activities of melatonin in intact and diseased gastrointestinal tract (git). J Physiol Pharmacol 2007;58:381-405.

28 Kennaway DJ, Wright H: Melatonin and circadian rhythms. Curr Top Med Chem 2002;2:199-209.

29 Reiter RJ: The pineal and its hormones in the control of reproduction in mammals. Endocr Rev 1980;1:109131.

30 Tan DX, Manchester LC, Terron MP, Flores LJ, Reiter RJ: One molecule, many derivatives: A never-ending interaction of melatonin with reactive oxygen and nitrogen species? J Pineal Res 2007;42:28-42.

31 Reiter RJ, Calvo JR, Karbownik M, Qi W, Tan DX: Melatonin and its relation to the immune system and inflammation. Ann N Y Acad Sci 2000;917:376-386.

32 Cao M, Wang P, Sun C, He W, Wang F: Amelioration of ifn-gamma and tnf-alpha-induced intestinal epithelial barrier dysfunction by berberine via suppression of mlck-mlc phosphorylation signaling pathway. PLoS One 2013;8:e61944. 